\title{
Leprosy elimination campaigns (LEC)
}

\section{Introduction}

The focus of the elimination strategy is to reduce the burden of disease to below one case per 10,000 population. In pursuit of this goal, WHO was able to obtain the necessary political commitment from governments, and through the support provided by various international and local non-governmental organizations, leprosy elimination activities were improved and strengthened. At the beginning of 1999 , more than 820,000 cases were registered for treatment in the world and around 805,000 new cases were detected during $1998 .{ }^{1}$

In most of the endemic countries the geographical coverage of multidrug therapy (MDT) services (diagnosis and treatment) is continuing to expand and this has resulted in an increased detection of new cases. In spite of tremendous progress made in eliminating leprosy in most of the high endemic countries, there are still areas where a significant number of cases remain undetected or where they are being detected late, i.e. at a stage where irreversible disabilities have occurred. In these areas the accessibility of health services, their capacity to provide MDT services and community awareness about the disease are still not at a satisfactory level.

In order to decrease the disease burden, these areas will need leprosy elimination campaigns (LEC) to reduce the pool of undetected cases in the community and cure them. These undetected (hidden) cases are individuals who have not been diagnosed and treated by the health services even though they have had signs and symptoms of leprosy for a considerable period of time. This could be because they do not know they have leprosy, or because they suspect or know they have leprosy but, because of the stigma, do not want to be identified as having the disease. Very often, such individuals do not know where to go for treatment.

\section{Objectives}

The main objective of LEC is to detect leprosy cases, particularly the more serious ones referred to as 'cases of consequence', that have remained undetected in the community, and to cure them with MDT. Leprosy of consequence is defined as cases with more than five skin lesions and skin smear positive cases (where facilities for skin smears are available and reliable). It is an initiative which aims at strengthening the ongoing leprosy elimination activities in carefully selected high endemic areas. ${ }^{2}$

Reproduced by kind permission of WHO, Action Programme for the Elimination of Leprosy, 20 Avenue Appia, CH-1211 Geneva 27, Switzerland. 


\section{Elements of LEC}

The strategy is based on a combination of three elements, namely: capacity building measures for local health workers to improve MDT services; increasing community participation so as to strengthen elimination activities at the local level; and diagnosing and curing patients. This basically is not a new concept as similar activities have been carried out by others in the field. However, under LEC several activities are grouped with the intention of obtaining maximum benefits by carrying them out in a systematic way.

Approaches taken to achieve the objectives vary from country to country, or even between regions within the same country, depending on the local situations and resources. The LEC is not intended to replace routine leprosy elimination activities but is aimed at strengthening and supplementing the ongoing activities.

\section{Activities}

The Campaigns first started out in areas with a population of around half-a-million. However, some countries modified this to cover large regions and populations because of the huge problem in hand and also because they have the money and manpower to carry them out. These large-scale campaigns mobilized several thousands of health workers and volunteers, and the case-finding activities were conducted within a period of one to two weeks. Currently, most of the high endemic countries are carrying out LEC as it was originally planned.

The main activities carried out under LEC are:

a) orientation workshops for local health workers and community volunteers;

b) community awareness creation and participation;

c) case-finding; and

d) treating every detected case with MDT and making efforts to ensure that each one is cured.

\section{A) ORIENTATION WORKSHOPS FOR LOCAL HEALTH WORKERS AND COMMUNITY VOLUNTEERS}

The aim of these workshops is to enable every health centre to provide MDT services to leprosy patients living within its area so as to improve accessibility. These workshops are expected to refresh the knowledge of the health workers and rekindle their interest in leprosy. In addition, the involvement of motivated and trained community health volunteers promoted greater community participation.

Such orientation workshops should be properly carried out so that the health workers' ability to provide MDT services are improved. Otherwise this will result in poor coordination between the local health workers, volunteers and the staff of the national programme and can cause problems of incorrect diagnosis and poor treatment completion.

\section{B) COMMUNITY AWARENESS CREATION AND PARTICIPATION}

This was done by informing the community leaders about the campaigns and ensuring their involvement in the various activities carried out in their community. In addition, before and during the time of the campaign, various forms of mass media were used to create greater 
awareness about leprosy, its curability and the availability of services for diagnosis and treatment. In the LECs that were carried out in certain selected districts, the use of mass media was limited (radio messages were broadcast in some cases) and community awareness was mainly promoted through village information meetings, poster displays and the distribution of pamphlets informing the public about leprosy and about the campaigns being conducted in their community. The large-scale campaigns used mass media more intensively, and this was in addition to the above mentioned village-level community awareness activities. The national programmes produced and broadcast various radio messages, interviews, TV spots and documentaries. Articles on leprosy were also published in the local newspapers.

Weakness in promoting community awareness might lead to poor community participation and low self-reporting of cases.

\section{C) CASE-FINDING}

The main thrust was on promoting self-reporting by individuals with suspicious signs/ symptoms to the campaign teams visiting villages/communities or to the health centre. By increasing community awareness, including through health education, individuals with obvious skin lesions become aware about the signs and symptoms of leprosy and selfreport for diagnosis. The community leaders and volunteers play a key role by assisting individuals with suspicious skin lesions to get themselves screened for leprosy. Ensuring that MDT services are available free of charge at the nearest health facility also increased the confidence of the community in the health services and in this way also promoted self-reporting.

Over-enthusiasm on the part of the health workers and volunteers, especially when targets for detections are being set, and the poor training received during the campaigns may result in over-diagnosis and in some instances already cured individuals could be re-registered as new cases.

\section{D) TREATING EVERY DETECTED CASE AND ENSURING CURE WITH MDT}

All new cases (including defaulters who needed treatment) were provided with the first dose of MDT at the time of diagnosis. Depending on the system of delivering MDT in the area, patients continued their treatment either at the nearest general health centre or at the specialized leprosy clinics. The health workers ensure that all patients put on treatment are able to complete the prescribed course within the specified time period, and all efforts are made to support patients in getting treatment regularly. Community volunteers mobilized during the campaigns played an important role in helping patients who for various reasons were not able to collect their MDT drugs regularly.

However, the limited health infrastructure in some areas will not be able to cope with the increased caseload. The collaboration between the specialized leprosy programme and the general health services may be limited and this will contribute towards patients not getting MDT drugs regularly.

\section{Outcome}

So far LECs have been conducted in 24 countries, covering a population of more than one 
billion. ${ }^{3-5}$ Since the start of the campaigns in 1995 , more than 500,000 cases were detected, of whom around 450,000 were detected in India alone. India carried out large-scale campaigns in 22 states during 1997-1998. Bangladesh and Nepal carried out similar large-scale campaigns during the early part of 1999.

The MB proportion of cases detected during the campaigns ranges from $32 \%$ in India to over $70 \%$ in Philippines and Sudan. The proportion of new cases with grade 2 disabilities ranges between $2 \%$ and $29 \%$ in Nigeria and Cambodia.

Apart from detecting cases, LECs carried out in Brazil, Madagascar, Nigeria, and Sudan were able to open new MDT clinics as part of their activities. These clinics are integrated into the existing primary health centres in the areas. Over 500,000 health workers and 300,000 volunteers participated in the campaigns. Because of the awareness generated by the campaigns a large number of defaulters were also brought back for treatment.

The LECs were able to generate a high degree of political commitment for elimination from authorities at various administrative levels. They also broadened the partnership for elimination by involving local NGOs and other community welfare organizations. Some of these local organizations contributed funds for posters and banners which were produced in local languages.

\section{Conclusion}

LECs were able to detect a large number of cases within a relatively short period of time and were able to treat them with MDT. The interest and support provided by the community as well as by the general health services were very high in all the campaigns. Because of the various mass media messages and information sessions conducted during the campaign, the public were made aware about the signs and symptoms and the availability of a cure free of charge for this disease which was at one time generally regarded as incurable.

\section{Acknowledgment}

Thanks are due to the national programme managers and to thousands of health workers and community volunteers for their various contributions. We are grateful to the Nippon Foundation for their generous contribution and the World Bank for their support in India and Bangladesh. Finally, we wish to thank the various local community organizations and our partners from the International Federation of Anti-Leprosy Associations (ILEP) for their help and support in making these campaigns successful.

\section{References}

1 World Health Organization. Global leprosy situation. Weekly Epidemiol Rec, 1999; 74: 313-316.

2 World Health Organization. Guidelines for carrying out Leprosy Elimination Campaigns, 1996, Action programme for the Elimination of Leprosy, CEE/CDS/WHO, 1996. (available on request from Leprosy Elimination Project)

3 World Health Organization. Leprosy elimination campaigns (LECs). Weekly Epidemiol Rec, 1998, 73: 177-184.

${ }^{4}$ World Health Organization. Leprosy elimination campaigns - reaching every patient in every village. Weekly Epidemiol Rec, 1997, 72: 205-212.

5 World Health Organization. Leprosy Elimination Campaigns-detecting and curing patients. Weekly Epidemiol Rec, 1999; 74: 329-334. 